\title{
Intelligent Navigation Oriented Mechanical Product CAD System Based on Knowledge Fusion
}

\author{
Yan $\mathrm{CaO}^{1,2, \text { a }}$, Zhongcan $\mathrm{Lin}^{2}$, Yonglou Zhao ${ }^{2}$, Changzhong Yuan ${ }^{2}$ and Yunfeng \\ Chen $^{2}$ \\ ${ }^{1}$ Xi'an Technological University, Xi'an, Shaanxi, China \\ ${ }^{2}$ Shaanxi Space Pump \& Valve Science and Technology Co.,Ltd, Xi'an, Shaanxi, China \\ a jantonyz@163.com
}

Keywords: Knowledge Fusion; Intelligent Navigation; Product Design; System Development.

\begin{abstract}
In order to improve intelligent heuristic performance, information support, computational capabilities, control mechanism, and interface self-adaptability of specialized product design systems, mechanical product design system based on knowledge fusion and intelligent navigation is studied systematically. After its product design flow is analyzed, the system is organized in terms of processes that constitute the whole design flow. Then, its information flow, control flow, and design flow are also determined. Based on knowledge fusion, the text of knowledge and information resources is defined that is the key basis and original information for system development. According to the requirements of CAD system development based on intelligent navigation, its architecture is put forward. Taking Oerlikon bevel gear transmission design for example, its functions and performance are illuminated in detail. The system can be applied to typical mechanical product design. While all-purpose CAD systems aim mainly at structure design, modeling and drafting, the developed system directs its attention to more specialized analyses and computation that make up for the deficiency of commercial CAD/CAM systems.
\end{abstract}

\section{Introduction}

Modern manufacturing puts forward new demands for CAD/CAM software. Traditional CAD/CAM software is usually all-purpose, but it does not provide enough specialized standards, criteria and fundamental data for product design in specific domains. As a result, design efficiency is not satisfying. At the same time, traditional CAD/CAM systems are mainly used to design product structures and not capable of professional analyses. It demands that professional CAD/CAM software provide various standards, criteria, analytic programs, fundamental data, and control mechanism. It makes designers devote themselves to creative thinking. This kind of CAD/CAM software embodies the thinking process of professionals and heuristic characteristics. In order to improve its adaptability, flexibility, and expediency, software developers must cooperate with professionals closely to develop product design system based on knowledge fusion and intelligent navigation [1,2].

Determination of knowledge-information resource text based on knowledge fusion. Knowledge-information resource text is determined by software experts and domain experts cooperatively based on the analysis of relevant technological data. It is the basis and original data for software development to determine whether the system developed can effectively realize intelligent navigation, heuristic design procedure, and knowledge fusion [3,4,5]. Its main contents are as follows:

- System functional and technological objectives.

- System resources and constraints.

- Product design flow and module partition based on process.

- Module definition and description.

- Interface standards.

- Data structure.

- Information structure.

- Formulas libraries. 
- Knowledge bases and Rule bases.

- Graph library.

- External and internal Databases.

- Information flow and control flow.

- Design result report generation.

- System test standards and test questions.

- etc.

Module partition based on courses. Based on the analysis of product design flow and process-based software structure, system module partitions keep to the following principles.

- A task should be independent of other tasks.

- Sequential tasks and concurrent tasks should be integrated.

- Tasks should have moderate granularity.

- A module's action region should be inside its control region.

- It is important to simplify the interface between modules.

- A module should have one input and one output to reduce the coupling between modules.

- A module's function can be predicted by a user, and its function should not be limited to a too low extent.

Determination of information flow, control flow and design flow. The essential of the whole design process is to accumulate, sort, process and output design information that flows among the modules. Not a module is completely independent with others. They are coordinated by design flow. The design flow and information flow interact with each other. After the design tasks are decomposed and the relation among them are analyzed, the design flow is formed that is represented in an intelligent navigation mode. The control flow reflects how a designer controls the design process. It adjusts and controls the design flow.

\section{Design system based on intelligent navigation}

Mechanical product design based on intelligent navigation and knowledge fusion is heuristic design $[6,7,8]$. In design process, the system guides users to make decision, choose parameters, and provide several data query mechanisms. Thus, it quickens the design process and makes it more efficient. According to the mode the system guides users, heuristic design can be divided into four categories.

- Parameter selection mode explains parameter selection principles that can be referred to by designers.

- Scope limit mode explains the permitted scope of the parameter that can be referred to by designers.

- In the design course, after initial conditions are input, rule database is activated, reasoning machine makes design choices according to initial conditions that will be confirmed by the designer finally.

- Hybrid heuristic mode is the combination of the aforementioned three modes. It possesses the merits of the three modes.

\section{System implementation}

The system is developed to design typical mechanical parts. Besides general developing requirements, such as structural layering, modulization, openness, expandablity, maintainability $[9,10]$, etc., CAD system based on intelligent navigation needs to coordinate the reciprocity of information flow, design flow, and control flow. It integrates design process control with information acquisition. Its architecture is shown in Fig.1.

\section{Oerlikon Bevel Gear Transmission Design System}

According to the above discussion, taking Oerlikon bevel gear transmission design system for 
example, its main functions and characteristics are illustrated as follows.

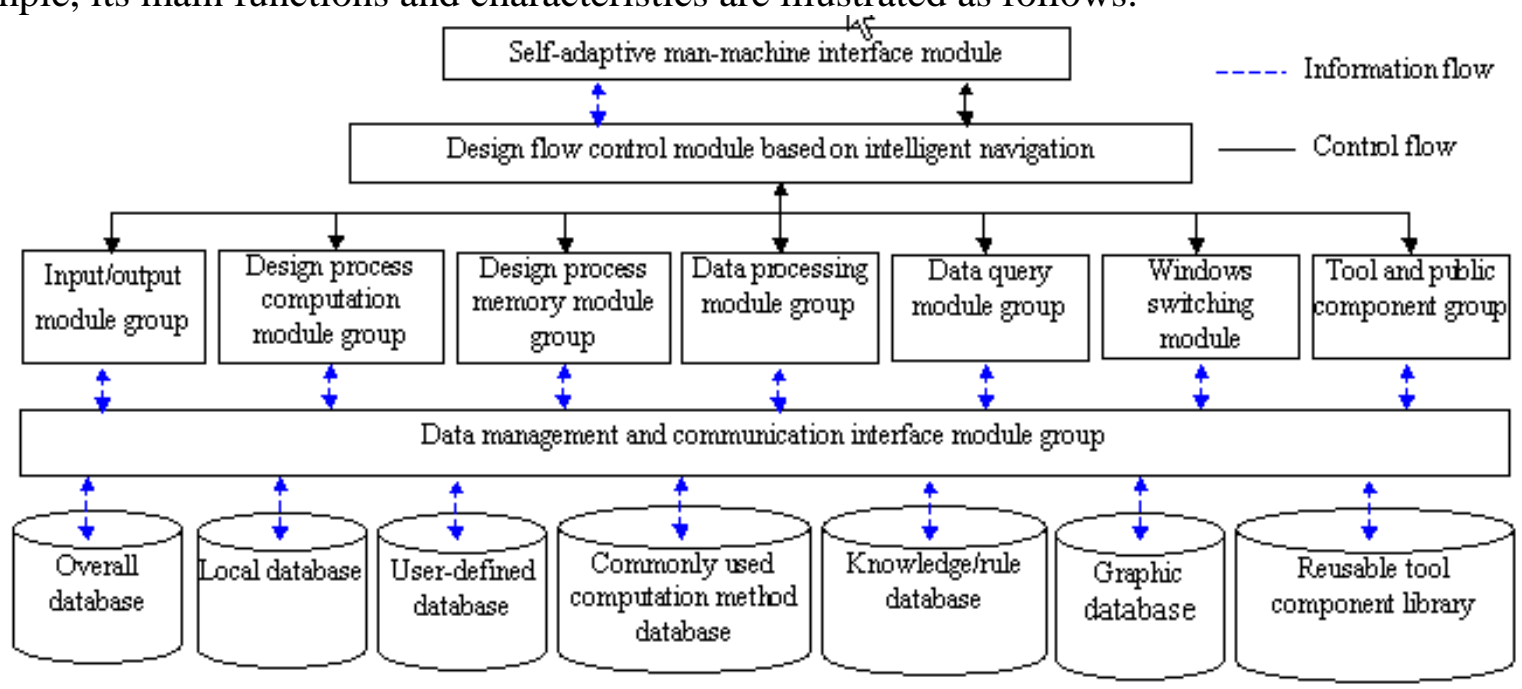

Fig.1. System architecture

The system is used to complete parameter input, computation, and strength check by calling LISP programs from AutoCAD. The system is divided into several design modules. The whole design process follows Oerlikon bevel gear transmission design flow, that is, parameter input, preliminary design, tooth surface contact strength check, dedendum flexural strength check, report generation, etc. It accords to designer's practical habits and is easy to use.

The preliminary design interface is shown in Fig.2. A user inputs design requirements and corresponding parameters that can be input or chosen by clicking a sideward button.

The design process accords strictly to the newest Mechanical Design Handbook. The main contents are to estimate the big-end reference diameter of a small gear. The parameters needed to be selected include gear material limits, transmission precision level, safety factor, and so on. All these parameters can be chosen from the charts and diagrams in the system.

Once all parameters are input, preliminary design results and many parameters that are needed in the latter modules are calculated. If the user does not complete the preliminary design and wants to go to the next design step, the system warns and prompts the user to complete the current computation.

After completing the preliminary design, the system automatically turns to Oerlikon bevel gear transmission design module, as shown in Fig.3. Based on the computation results, the system prompts the user to manually determine a appropriate value that satisfy some requirements. This can assure the correctness of the final design results.

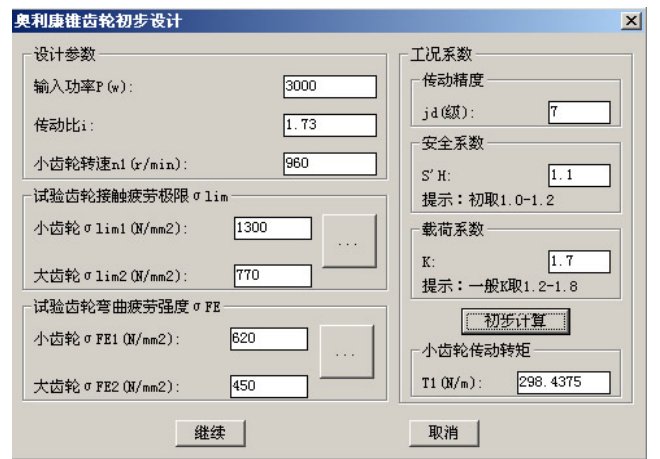

Fig.2. Preliminary design interface

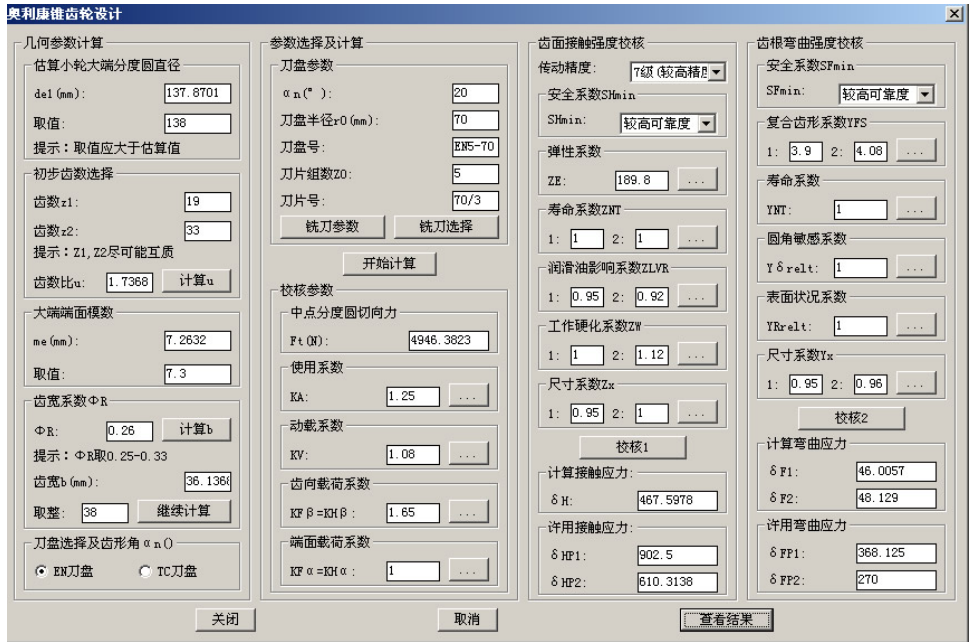

Fig.3. Oerlikon bevel gear transimission design interface

After all the parameters are input, the system returns the calculation results. If the results are not satisfying, the Oerlikon bevel gear transmission needs to be redesigned. Otherwise, go to the next step. After completing the whole Oerlikon bevel gear transmission design process, the system generates a design result report. 


\section{Conclusions}

According to the design cycle of Oerlikon bevel gear transmission, using navigation patterns, a designer is guided to accomplish its design, calculation, check and parametric drawing step by step according to its performance requirements. With the development tools of Visual LISP, module programming is used to develop the Oerlikon bevel gear transmission CAD system. The system includes transmission design process control module, algorithm module, parameter data module and parametric drawing module. The man-machine interface is developed using Dialog Control Language according to engineering personnel's habits. Design parameters are digitalized to establish parameter databases. The system is intuitionistic and easy to use, and satisfies the requirements of gear transmission design in mechanical industries. Consequently, Oerlikon bevel gear design flow is standardized and its design efficiency and quality can be improved. The approach can also be applied to develop other professional gear transmission design systems in special domains.

\section{Acknowledgments}

The paper is supported by Special Scientific Research Project of Shaanxi 13115 Scientific and Technological Innovation Engineering Project (2010FWPT-05), 2011 Key Education Innovation Project of Xi'an Technological University (11JGZ02), Shaanxi Provincial Department of Education (09JK475), Shaanxi Major Subject Construction Project and President Scientific Research Fund of Xi'an Technological University.

\section{References}

[1] R. S. Amant, T. Long and M. S. Dulberg: Knowledge-Based Systems Vol. 11 (1998), p. 61-70

[2] X. Q. Wang, X. L. Wang and R. J. Zhao: Mini-Micro System Vol. 21 (2000), p. 475-477

[3] P. O'Grady and W. Y. Liang: Computer Integrated Manufacturing Systems Vol. 11 (1998), p. 267-283

[4] Y. Cao, R. J. Zhao and Z. Q. Liu: Mini-Micro System Vol. 25 (2004), p. 2277-2281

[5] H. Q. Ni, Y. C. Zhao, P. Luo, J. P Zhang and J. L. Bai: Heavy Machinery Science and Technology No. 3 (2004), p. 13-5

[6] Y. T. Lei and Z. J. Yang: Journal of Machine Design Vol. 24 No. 3 (2007), p. 57-60

[7] D. F. Ren, X. Z Deng and X. Z. Ren: Machinery Design \& Manufacture No. 2 (2002), p. $13-15$

[8] H. T. Li and W. J. Wei: Journal of China Agricultural University Vol. 9 No. 5 (2004), p. 45-50. 